\title{
USO DE ÁCIDOS ORGÁNICOS PARA MEJORAR LOS PARÁMETROS ZOOTÉCNICOS Y LA CALIDAD DE LA CARCASA DE POLLOS DE ENGORDE
}

AUTORES: Karen Pilar Quijije Quiroz ${ }^{1}$

Omelio E. Borroto Leal ${ }^{2}$

Hilda Brigitte Quijije Quiroz ${ }^{3}$

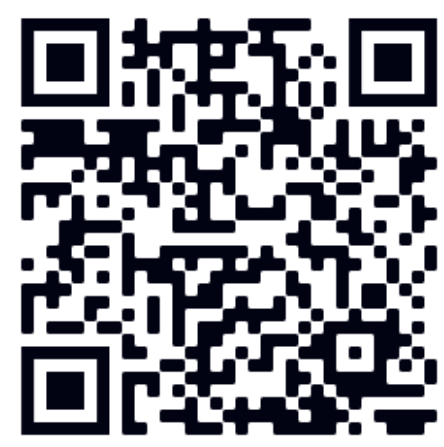

DIRECCIÓN PARA CORRESPONDENCIA:karenpili1906@hotmail.com

Fecha de recepción: 02/11/2019

Fecha de aceptación: 01/12/2019

\section{RESUMEN}

El presente trabajo sobre el uso de ácidos orgánicos para mejorar los parámetros productivos zootécnicos y la calidad de la carcasa de los pollos de engorde se desarrolló en el sitio Andil zona rural del Cantón Jipijapa a 4,0 km aproximadamente por la vía Universitaria Noboa - Jipijapa, cuya finalidad fue establecer la dosis adecuada de ácidos orgánicos en la producción de pollos de engorde según el sexo (mortalidad, consumo de alimento, ganancia de peso y conversión alimenticia), evaluar la calidad de la carcasa de acuerdo a lesiones presentes en órganos respiratorio (pulmón y sacos aéreos) y digestivos (intestino y riñón), determinar mediante un análisis benefició costo la dosis rentable para la producción avícola. Dentro del desarrollo de la investigación se abordaron los diferentes métodos científicos tales como métodos teóricos: histórico-lógico, análisis-síntesis; método estadístico-matemático y el método bibliográfico. Los resultados permiten concluir que la mortalidad tuvo un porciento bajo con una muerte de 2 pollos en el T8, en cuanto a consumo de alimento el T6 M fue el más eficiente, ganancia de peso diferencia estadística en T4 M, demostró mayor eficiencia comparada al resto de tratamientos, la conversión alimenticia obtuvimos alta significancia estadística en el T3 H, con 1,92 siendo más eficiente la hembra.

PALABRAS CLAVE: alimenticia; conversión; producción; significancia

\section{ACADEMIC LIVES OF THE STUDENTS OF THE FIRST SEMESTER MATUTINE OF INFORMATION TECHNOLOGIES-UNESUM}

\footnotetext{
1 Ingeniera Agropecuaria Universidad Estatal del Sur de Manabí. Jipijapa-Ecuador. Email: karenpili_1906@hotmail.com

${ }^{2}$ Ingeniero agrónomo; PhD en Administración. Docente y Rector de la Universidad Estatal del Sur de Manabí. Presidente de la Red Ecuatoriana Universitaria de Vinculación con la Comunidad (REUVIC). Email:omelio.borroto@unesum.edu.ec

${ }^{3}$ Estudiante de la Universidad Estatal del Sur de Manabí. Jipijapa-Ecuador. Email: hildab_1999@hotmail.com
}

(C) Universidad Estatal del Sur de Manabí. Jipijapa, Ecuador. 
Karen Pílar Quijije Quiroz, Omelio E. Borroto Leal, Hilda Brigitte Quijije Quiroz

\section{ABSTRACT}

The present work on the use of chemical parameters to improve the zootechnical production parameters and carcass quality of broilers was developed in the Andil site in rural area of Jipijapa Canton, at approximately $4 \mathrm{~km}$ by University - Jipijapa rood Noboa. Chose purpose was to establishing the appropriate dose of organic acid in the production of broiler chickens according to sex (mortality, food consumption, weight gain and feed conversion), evaluate the quality of the carcass according to lesions present in respirators organs (lung and sacs) and digestion (intestine and kidney). Determine through and analysis, benefit, cast the profitable dose. Within the development of the research, different scientific methods such as theoretical methods involving historical-logic, analysis-synthesis, mathematical statistical method and bibliographic method were addressed. The results conclude that the cloud had a low $0 \%$ with a death of 2 chickens in T8, in terms of food consumption the T6 $\mathrm{M}$ was the most efficient, weight gain statistical difference in T4 M, showed greater efficiency compared to the rest of the treatment, high feed conversion to high statistical significance in $\mathrm{T} 3 \mathrm{H}$, with 1.92 being more efficient female.

KEYWORDS: food conversion; production; significance

\section{INTRODUCCIÓN}

Desde 1992, el consumo de carne de aves se incrementó en el Ecuador de 7,5 kilos por persona al año a 32 kilos hasta 2011, mientras que los huevos subieron de 32 unidades a 140, consumo per cápita en el mismo periodo. La producción local en la actualidad satisface toda la demanda de pollos y huevos del INEC sobre superficie y producción agropecuaria continua, el número de aves criadas en planteles avícolas entre el 2010 y 2011 subió 7,99\%. Si bien no hay información actualizada sobre la cantidad de productores de aves en el país, el último censo realizada en 2006 reveló que a esa fecha existían 1567 productores, entre pequeños medianos y grandes (Trujillo, 2016)

Uno de los principales objetivos en la industria avícola es lograr mayor rapidez en el crecimiento y la capacidad de engorde de los animales, para lo cual se han seguido varias estrategias. Una de ellas, el uso de antibióticos como promotores de crecimiento. (Medina N. M., 2014).

La carne de pollo es un alimento con una alta densidad de nutrientes y baja densidad energética y no solo son de especial relevancia en la dieta de la población en general, sino también y especialmente en algunos grupos como ancianos, adolescentes, gestantes, personas que realizan dietas hipocalóricas, entre otros (Carbajal, 2005).

La acción de los ácidos orgánicos sobre la microflora intestinal se lleva a cabo mediante dos mecanismos: (a) reduciendo el $\mathrm{pH}$ del alimento y del tracto digestivo, creando un entorno negativo para el crecimiento de microorganismos patógenos de los géneros Escherichia, Clostridium y Salmonella; y (b) el efecto antimicrobiano específico debido a la forma no disociada, alterando varios procesos esenciales para la vida de los microorganismos, principalmente Gram negativos (Gonzáles, 2013).

En la zona sur de Manabí esta industria es muy importante por ser una actividad que representa ingresos económicos, pero los costos de producción son elevados por el uso de antibióticos en la vida de las aves hasta el sacrificio, poniendo en riesgo la salud de los consumidores finales,

44 UNESUM-Ciencias. Publicación cuatrimestral. Vol. 3, Año 2019, No. 3 (Septiembre - Diciembre) 
generando de forma inconsciente con este manejo poco saludable. La producción avícola actual tiene como retos importantes, garantizar la seguridad alimentaria, proteger el medio ambiente y buscar el máximo bienestar para las aves, ajustándose para ello a la legislación vigente en estos temas; pero sin perder de vista los dos objetivos primordiales que le permitirán sobrevivir en el mercado y ser competitiva.

\section{DESARROLLO}

\section{Materiales y métodos}

Para la implementación del experimento, se contó con 432 pollos (216 machos y 216 hembras) estirpe Cobb 500, las medidas del galpón fueron de 10 X 20 metros, 24 comederos y 24 bebederos, los cuales permitieron controlar de mejor manera el consumo de alimento y agua, los materiales fueron los siguientes:

- Criadoras a gas

- Termómetros

- Malla de alambre electro soldadas

- Bebederos

- Comederos

- Cortinas

- Viruta o tamo de arroz

- Alimentos

- Ácidos orgánicos

- Jeringas

- Bombas de fumigar

- Balanza

- Medicamentos

- Registros

- Gas 


\section{Ubicación}

La investigación se realizó en la Granja Experimental Andil el cual tuvo una duración de los meses de julio a agosto 2019 .

\section{Factores en estudio}

Factor A

- Dosis de ácidos orgánicos

Factor B

- Sexo de aves

\section{Tratamientos}

Los tratamientos que se evaluaron en la investigación fueron las siguientes:

Cuadro 2. Tratamientos en estudio

\begin{tabular}{llll} 
TRATAMIENTO & CODIGO & FACTOR A & FACTOR B \\
\hline 1 & D0S1 & $3,6 \mathrm{ccL}$ Oxitetraciclina & Hembra \\
2 & D0S2 & $3,6 \mathrm{cc} / \mathrm{L}$ Oxitetraciclina & Macho \\
3 & D1S1 & $1,0 \mathrm{cc} / \mathrm{L}$ Ácido Orgánico & Hembra \\
4 & D1S2 & $1,0 \mathrm{cc} / \mathrm{L}$ Ácido Orgánico & Macho \\
5 & D2S1 & $2,0 \mathrm{cc} / \mathrm{L}$ Ácido Orgánico & Hembra \\
6 & D2S2 & $2,0 \mathrm{cc} / \mathrm{L}$ Ácido Orgánico & Macho \\
7 & D3S1 & $3,0 \mathrm{cc} / \mathrm{L}$ Ácido Orgánico & Hembra \\
8 & D3S2 & $3,0 \mathrm{cc} / \mathrm{L}$ Ácido Orgánico & Macho \\
\hline
\end{tabular}

Elaboración propia.

Fuente: Investigación de campo.

\section{Diseño experimental}

El diseño experimental utilizado fue bloque completamente al azar con arreglo factorial 4X2. Cuyo modelo matemático lineal es:

Yijk $=\mu+\beta i+d j+d k+d d j k+e j k l$

\section{Análisis estadístico}

\section{Esquema del análisis de varianza}

Cuadro 3. Esquema del análisis de varianza

\section{Fuente de variación}

Grados de libertad 


\begin{tabular}{ll}
\hline Repeticiones & $2(\mathrm{r}-1)$ \\
Tratamientos & $7(\mathrm{t}-1)$ \\
Dosis & $3(\mathrm{D}-1)$ \\
Sexo & $1(\mathrm{~S}-1)$ \\
Interacción D x S & $3(\mathrm{DxS})$ \\
Testigo vs resto & 1 \\
Error Experimental & $14\{$ T-r-t-(ts vs rs $)\}$ \\
\hline Total & $\mathbf{2 3}$ \\
\hline
\end{tabular}

Elaboración propia.

Fuente: Investigación de campo.

\section{Variables a ser evaluadas}

Para el desarrollo del experimento, se consideraron las siguientes variables por objetivos.

Objetivo. 1 Establecer la dosis adecuada de ácido orgánico en la producción de pollos de engorde según el sexo.

Índice de mortalidad (\%): es el porcentaje de aves muertas en un lapso determinado.

$$
\text { Indice de mortalidad }=\frac{\text { aves muertas en el periodo } \times 100}{\text { Animales al empezar el periodo }}
$$

Índice de conversión (IC): es una característica heredable y fácilmente afectada por el alimento de baja calidad, enfermedades y mal manejo.

$$
I C=\frac{\mathrm{kg} \text { alimento consumido }}{\text { Peso vivo } \mathrm{kg}}
$$

Consumo de alimento diario (promedio semanal) por ave (CADA): Para obtener el CADA, el resultado se divide entre el numero promedio de aves en la semana.

Consumo acumulado por (unidad experimental): es la suma del consumo de alimento total dividida por el número de aves existente ave.

Ganancia de peso diario (promedio semanal) (GPD): es el promedio de peso por ave.

Objetivo. 2 Evaluar la calidad de carcasa de acuerdo a lesiones presente en órganos respiratoria y digestivos.

Para el cumplimiento del objetivo 2, se realizó la necropsia aviar, la cual consistió en lo siguiente:

Para la necropsias se sacrificó 1 ave semanalmente en cada unidad para evaluar la calidad de la canal, el monitoreo fue constante y si de presentarse un problema de lesión se hizo sacrificios para conocer las causa o identificar la patología presente en los lotes para realizar el tratamiento correspondiente de igual manera a la aves muerta de forma natural también se les realizo la necropsia.

Cuadro 5. Necropsias aviar 
Karen Pilar Quijije Quiroz, Omelio E. Borroto Leal, Hilda Brigitte Quijije Quiroz...

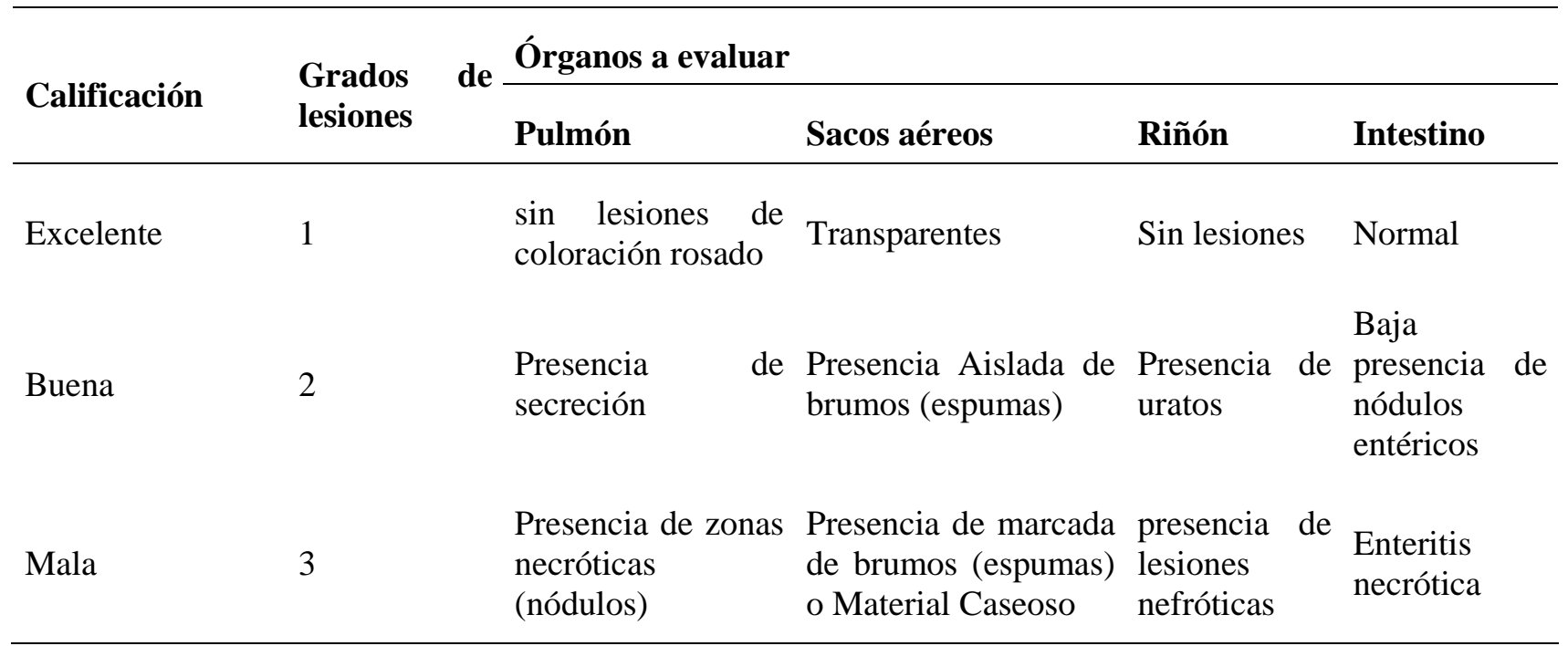

Elaboración propia.

Fuente: Investigación de campo.

Objetivo. 3 contribuyó a determinar mediante un análisis beneficio costo la dosis rentable para la producción avícola.

\section{Análisis Económico}

Para evaluar la conveniencia económica de la utilización de ácidos orgánicos; se realizó un análisis económico a través del enfoque costo beneficio.

\section{RESULTADOS Y DISCUSIÓN}

Los resultados obtenidos de la investigación se presentan por objetivos tal como se describen a continuación:

En lo que respecta al objetivo 1 "Establecer la dosis adecuada de ácido orgánico en la producción de pollos de engorde según el sexo", se consideraron las siguientes variables: mortalidad, índice de conversión, consumo de alimento diario y ganancia de peso a partir del promedio semanal, a partir de cada unidad experimental; acciones que se consideraron oportunas en la determinación de la dosis adecuada de ácidos orgánicos en la producción de pollos de engorde. Es oportuno mencionar que el estudio contempló la respuesta de los pollos en función a su sexo.

Se presenta los resultados obtenidos en el siguiente orden

\section{Mortalidad}

Cuadro 6. Mortalidad por tratamientos

\begin{tabular}{ll}
\hline TRATAMIENTO & MORTALIDAD \\
\hline $\mathrm{T} 1$ & 0 \\
$\mathrm{~T} 2$ & 1 \\
$\mathrm{~T} 3$ & 0 \\
$\mathrm{~T} 4$ & 0
\end{tabular}




\begin{tabular}{ll}
$\mathrm{T} 5$ & 1 \\
$\mathrm{~T} 6$ & 0 \\
$\mathrm{~T} 7$ & 0 \\
\hline $\mathrm{T} 8$ & 2 \\
\hline
\end{tabular}

Elaboración propia.

Fuente: Investigación de campo.

El porcentaje de mortalidad durante el experimento tuvo niveles bajos; en cuanto a los porcentajes de mortalidad fueron para el Ex1 de 0\%; para el grupo Ex 2 de 0\%; y para el grupo Testigo de $1,67 \%$ concluyendo de esta manera con un producción muy satisfactoria y rentable para el productor avícola.

\section{Ganancia de peso}

$\mathrm{El}$ análisis de varianza efectuado sobre la variable ganancia de peso (cuadro $\mathrm{N}^{\circ} 7$ ), presenta diferencias estadísticas altamente significativas para tratamientos y sexo, lo que determina variabilidad entre sexo y tratamientos de manera independiente, las otras fuentes de variaciones no presentan ninguna diferencia estadística. El coeficiente de variación que se presenta es 6,58.

Cuadro 7. Cuadrados medios de ganancia de peso

\section{Cuadro de Análisis de la Varianza (SC tipo I)}

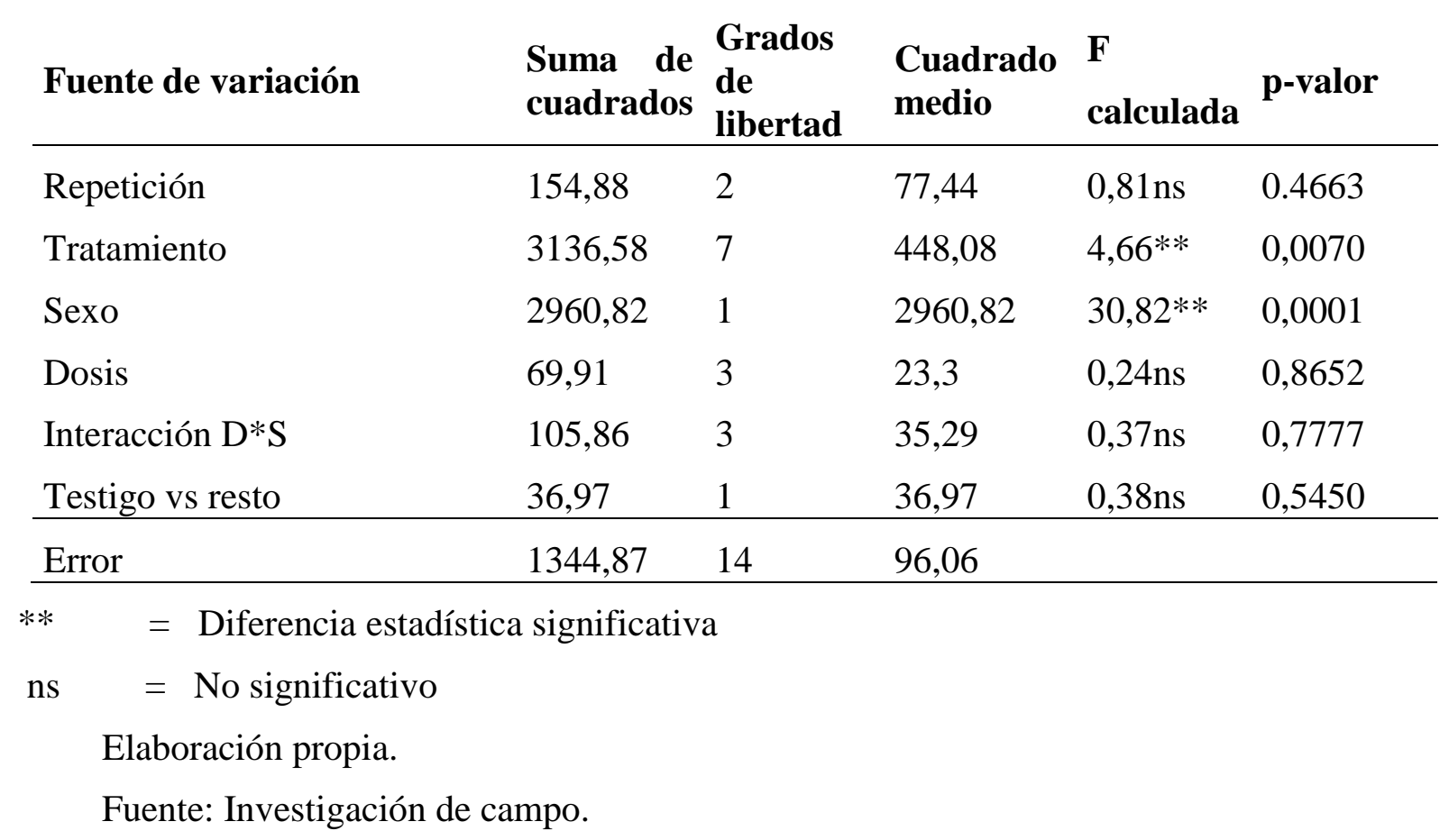

Con el fin de establecer el o los mejores tratamientos, se realizó la prueba de Duncan al 0,05\% de probabilidades (cuadro 8), observándose significancia a nivel de tratamientos, determinándose como mejores, los tratamientos con ácido orgánicos en machos. Donde se observa mejores resultados en los tratamientos 4, 6 y 8, todos con aplicación de ácidos orgánicos, 1, 2 y 3 cc respectivamente. 
Karen Pilar Quijije Quiroz, Omelio E. Borroto Leal, Hilda Brigitte Quijije Quiroz...

Cuadro 8. Prueba de Duncan para los tratamientos

\begin{tabular}{llllllll}
\hline Tratamiento & medias & N & E.E & \multicolumn{3}{c}{ Resultados } \\
\hline 1,0 cc/L Ácido Orgánico T4 M & 164,30 & 3 & 5,66 & A & & \\
2,0 cc/L Ácido Orgánico T6 M & 161,35 & 3 & 5,66 & A & & \\
3,0 cc/L Ácido Orgánico T8 M & 159,62 & 3 & 5,66 & A & & \\
3,6 cc/L Oxitetraciclina T2 M & 155,12 & 3 & 5,66 & A & B & \\
2,0 cc/L Ácido Orgánico T5 H & 140,69 & 3 & 5,66 & & B & C \\
3,6 cc/L Oxitetraciclina T1 H & 138,56 & 3 & 5,66 & & B & C \\
1,0 cc/L Ácido Orgánico T3 H & 136,22 & 3 & 5,66 & & & C \\
\hline 3,0 cc/L Ácido Orgánico T7 H & 136,07 & 3 & 5,66 & & & C \\
\hline
\end{tabular}

Elaboración propia.

Fuente: Investigación de campo.

Cuadro 9. Análisis de resultados de prueba de Duncan a nivel de sexo.

\begin{tabular}{ccccc}
\hline Sexo & Medias & N & E.E & Resultado \\
\hline M & 160,10 & 12 & 2,79 & A \\
H & 137,89 & 12 & 2,79 & B \\
\hline Elaborado por: Karen Quijije & & & \\
Fuente: Investigación de campo & &
\end{tabular}

De igual manera se realiza la prueba de significación de Duncan a nivel de sexo, a fin de establecer diferencia estadísticas, determinándose que hubo mejor respuesta al ácido orgánico con respecto a ganancia de peso, en los machos, tal como se aprecia en el gráfico dos.

Es oportuno mencionar que los datos fueron tomados semanalmente, lo que motivo la realización previa de un análisis de tendencia (gráfico $\mathrm{N}^{\circ} 1$ ), que permitió observar la variabilidad semanal del peso por tratamientos, por sexo y por dosis, situación que se realizó con respecto a la ganancia de peso, consumo de alimento y conversión alimenticia. El siguiente grafico expresa el procedimiento realizado a fin de filtrar los datos en un análisis integral de la investigación, definiendo una respuesta no de la última semana sino de todo el proceso investigativo.

Gráfico 1. Análisis de tendencia semanal de Ganancia de peso 


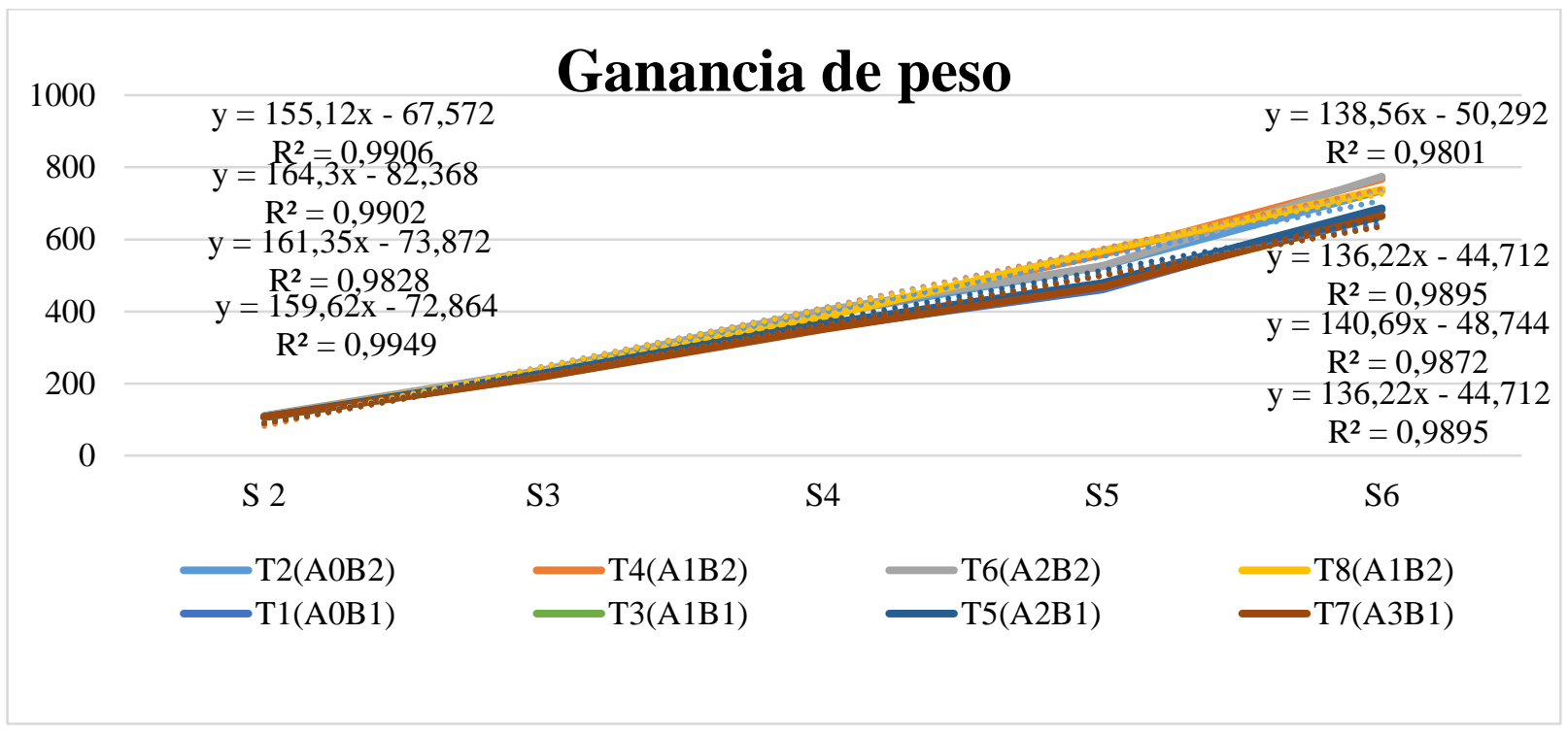

Elaboración propia.

Fuente: Investigación de campo.

En sexo la diferencia estuvo en los machos, (Rogel, 2014), sobre el incremento de peso se infiere que el tratamiento dos dio mejor resultado, con acidomix, pues el uso de ácidos orgánicos demostró mejorar la salud del tracto gastrointestinal y fue clave en la productividad de los animales, el peso corporal y la ganancia de peso de las aves al final del estudio fue estadísticamente similar entre los tres tratamientos. Estas diferencias entre estudios pudieron deberse al tipo y dosis de ácido orgánico empleado.

\section{Consumo de alimento}

Para el análisis de la variable consumo de alimento, se realizó su respectivo análisis de tendencia, y de este análisis integral se obtuvieron los siguientes resultados: se presentó diferencia estadística a nivel de tratamiento con un p-valor 0,050, a nivel de sexo con p-valor 0,0054 se determina diferencia estadística altamente significativa, las otras fuentes de variación tales como; interacción dosis - sexo, y testigo versus el resto no presentaron diferencias estadísticas (Cuadro 10). El coeficiente de variación fue de 3,32.

Cuadro 10. Cuadrados medios de consumo de alimento

Cuadro de Análisis de la Varianza (SC tipo I)

\begin{tabular}{llllll}
\hline \multicolumn{1}{c}{ F.V. } & $\mathrm{SC}$ & $\mathrm{gl}$ & $\mathrm{CM}$ & $\mathrm{F}$ & p-valor \\
\hline Repetición & 248,69 & 2 & 124,34 & $1,44 \mathrm{~ns}$ & 0,2691 \\
Tratamiento & 1661,48 & 7 & 237,35 & $2,76^{*}$ & 0,0505 \\
Sexo & 931,88 & 1 & 931,88 & $10,82^{* *}$ & 0,0054 \\
Dosis & 388,09 & 3 & 129,36 & $1,50 \mathrm{~ns}$ & 0,2573 \\
Interacción D*S & 341,51 & 3 & 113,84 & $1,32 \mathrm{~ns}$ & 0,3068 \\
Testigo vs resto & 221,52 & 1 & 221,52 & $2,57 \mathrm{~ns}$ & 0,1311 \\
\hline Error & 1205,66 & 14 & 86,12 & & \\
\hline
\end{tabular}


Karen Pilar Quijije Quiroz, Omelio E. Borroto Leal, Hilda Brigitte Quijije Quiroz...

$$
\begin{array}{ll}
* * & =\text { Diferencia estadística significativa } \\
* & =\text { Diferencia estadística significativa } \\
\mathrm{ns} & =\text { No significativo }
\end{array}
$$

Elaboración propia.

Fuente: Investigación de campo.

Cuadro 11. Prueba de Duncan consumo de alimento para los tratamientos

\begin{tabular}{lllllll}
\hline Tratamiento & Medias & n & \multicolumn{2}{c}{ E.E } & \multicolumn{2}{c}{ Resultados } \\
\hline 2,0 cc/L Ácido Orgánico T6 M & 296,57 & 3 & 5,66 & & A & \\
3,0 cc/L Ácido Orgánico T8 M & 286,16 & 3 & 5,66 & A & B \\
1,0 cc/L Acido Orgánico T4 M & 282,09 & 3 & 5,66 & A & B \\
1,0 cc/L Ácido Orgánico T3 H & 277,35 & 3 & 5,66 & A & B \\
3,6 cc/L Oxitetraciclina T2 M & 276,84 & 3 & 5,66 & A & B \\
2,0 cc/L Ácido Orgánico T5 H & 273,01 & 3 & 5,66 & A & B \\
3,6 cc/L Oxitetraciclina T1 H & 271,01 & 3 & 5,66 & A & B \\
\hline 3,0 cc/L Ácido Orgánico T7 H & 269,78 & 3 & 5,66 & & B \\
\hline
\end{tabular}

Elaboración propia.

Fuente: Investigación de campo.

Cuadro 12. Prueba de Duncan para consumo de alimento para sexo

\begin{tabular}{lllll}
\hline Sexo & Medias & $\mathrm{n}$ & E.E & Resultado \\
\hline $\mathrm{M}$ & 285,42 & 12 & 2,75 & $\mathrm{~A}$ \\
$\mathrm{H}$ & 272,95 & 12 & 2,75 & $\mathrm{~B}$ \\
\hline
\end{tabular}

Elaboración propia.

Fuente: Investigación de campo.

El Cuadro 12, presenta la prueba de Duncan realizada al 0,05\% de probabilidades, se puede observar que el T6 presenta una mejor respuesta al ácido orgánico, con la dosis de $2 \mathrm{~cm}$, seguido del T8 con $3 \mathrm{~cm}$ de ácido orgánico.

En sexo la mayor representatividad de consumo de alimento está en el macho.

Para el consumo de alimento el T6 fue diferente el resto de tratamientos tuvieron consumos similares, esto es fundamentado por (Rogel, 2014) quien dice No existe información acerca de la utilización de ácidos orgánicos en pollos finqueros pero por similitud fisiológica intestinal, no así en su velocidad de crecimiento pueden comportarse de igual manera que en el boiler promoviendo una mejora en la microflora intestinal.

\section{Conversión alimenticia}

El cuadro 13, representa el análisis de varianza efectuado en el que se puede notar que si existen diferencias estadísticas significativas y altamente significativas en tratamientos y en sexo 
respectivamente, las otras fuentes de variaciones tales como interacción dosis sexo y testigo versus resto, no presentaron ninguna diferencia estadística. El coeficiente de variación que se presenta es 11,77 .

Se destaca que el análisis fue realizado considerando los datos tomados semana a semana, se empleó para tal efecto el análisis de tendencia, mismo que permitió observar la curva semanal del proceso de crecimiento de los pollos de manera integral.

Cuadro 13. Cuadrados medios de conversión alimenticia

Cuadro de Análisis de la Varianza (SC tipo I)

\begin{tabular}{llllll}
\hline F.V. & SC & gl & CM & F & p-valor \\
\hline Repetición & $3,90 \mathrm{E}-03$ & 2 & $1,40 \mathrm{E}-03$ & $1,44 \mathrm{~ns}$ & 0,2691 \\
Tratamiento & 0,01 & 7 & $1,20 \mathrm{E}-03$ & $2,76^{*}$ & 0,0505 \\
Sexo & 0,01 & 1 & 0,01 & $31,25^{* *}$ & 0,0001 \\
Dosis & $3,30 \mathrm{E}-04$ & 3 & $1,10 \mathrm{E}-04$ & $0,34 \mathrm{~ns}$ & 0,7942 \\
Interacción D*S & $3,70 \mathrm{E}-04$ & 3 & $1,20 \mathrm{E}-04$ & $0,38 \mathrm{~ns}$ & 0,7724 \\
Testigo vs resto & $8,40 \mathrm{E}-05$ & 1 & $8,40 \mathrm{E}-05$ & $0,26 \mathrm{~ns}$ & 0,6151 \\
\hline Error & $4,50 \mathrm{E}-03$ & 14 & $3,20 \mathrm{E}-04$ & & \\
\hline
\end{tabular}

** $\quad=$ Diferencia estadística significativa

* $\quad=$ Diferencia estadística significativa

ns $\quad=$ No significativo

Elaboración propia.

Fuente: Investigación de campo.

En función a los resultados obtenidos, se efectuó la prueba la de Duncan para tratamientos y sexo, mismos que a continuación se presentan.

Cuadro 14. Prueba de Duncan en tratamientos de conversión alimenticia.

\begin{tabular}{llllllll}
\hline Tratamiento & Medias & n & E.E & & \multicolumn{2}{c}{ Resultados } \\
\hline 1,0 cc/L Ácido Orgánico T3 H & 0,18 & 3 & 0,01 & & A & & \\
3,0 cc/L Ácido Orgánico T7 H & 0,17 & 3 & 0,01 & A & B & \\
3,6 cc/L Oxitetraciclina T1 H & 0,17 & 3 & 0,01 & A & B & \\
2,0 cc/L Ácido Orgánico T5 H & 0,16 & 3 & 0,01 & A & B & C \\
2,0 cc/L Ácido Orgánico T6 M & 0,14 & 3 & 0,01 & B & C & \\
1,0 cc/L Ácido Orgánico T4 M & 0,13 & 3 & 0,01 & B & C & \\
3,0 cc/L Ácido Orgánico T8 M & 0,13 & 3 & 0,01 & C & & \\
\hline 3,6 cc/L Oxitetraciclina T2 M & 0,13 & 3 & 0,01 & & C & & \\
\hline
\end{tabular}

Elaboración propia.

Fuente: Investigación de campo. 
Karen Pilar Quijije Quiroz, Omelio E. Borroto Leal, Hilda Brigitte Quijije Quiroz...

Se observa en el cuadro 14, que existe mejor respuesta a nivel de conversión alimenticia en las hembras, siendo el mejor tratamiento el de 1cc de ácido orgánico, seguido en todo caso por el resto de tratamientos con hembras. Lo que se ratifica en la prueba de Duncan en sexo, expuesto en el siguiente cuadro

Cuadro 15. Prueba de Duncan en sexo de conversión alimenticia.

\begin{tabular}{lllll}
\hline Sexo & Medias & N & E.E & Resultados \\
\hline $\mathrm{H}$ & 0,17 & 12 & 0,01 & $\mathrm{~A}$ \\
$\mathrm{M}$ & 0,13 & 12 & 0,01 & $\mathrm{~B}$ \\
\hline
\end{tabular}

Elaboración propia.

Fuente: Investigación de campo.

Es evidente dada las pruebas realizadas, que las hembras convierten de mejor manera el alimento que consumen, y que el ácido orgánico pudo haber influido en su mejor asimilación del alimento, el grafico 6 refleja las diferencias a nivel de promedios a favor de las hembras.

Para la conversión alimenticia el valor general es 1,79, esto es corroborado por lo indicado por (TRUJILLO, 2016) en cuanto a conversión alimenticia total se notan diferencias altamente significativas entre los tratamientos sometidos al experimento con un promedio general de $1,90 \mathrm{y}$ a esto los resultados obtenidos por (Rogel, 2014) afirma que en la investigación, son similares a estudios realizados por otros autores con la inclusión de ácidos orgánicos, siendo el tratamiento dos y uno los de mejores resultados (3.13 y 3.30 la conversión alimenticia respectivamente; sin ofrecer significación estadística entre tratamientos $(\mathrm{p}<0.05)$.

\section{Para cumplir el objetivo 2, se realizó la necropsia aviar, la cual consistió en lo siguiente:}

Para la necropsia se sacrificó un ave quincenalmente en cada unidad experimental y se evaluó la calidad de la canal, el monitoreo que se hizo fue constante pero no se presentaron lesiones, por lo que se puede decir que se obtuvo un pollo con excelente calificación en el ensayo. Destacando el hecho que a este nivel no se establece diferencia estadística entre los tratamientos; de igual manera en lo que respecta al sexo no se determinan diferencias en cuanto a calidad de canal por el uso o no de ácidos orgánicos.

En el cuadro 16, se pueden observar los parámetros que se evaluaron con resultados que tienden a la excelencia, de igual forma en los anexos se reflejan que no presentaban ninguna patología mediante la necropsia.

Cuadro 16 . Representatividad de la necropsia aviar

\begin{tabular}{lllllll}
\hline Tratamientos & & Grados & \multicolumn{2}{l}{ Órganos a evaluar } \\
\cline { 5 - 7 } $\begin{array}{l}\text { Dosis } \\
\text { Sexo }\end{array}$ & Calificación & $\begin{array}{l}\text { de } \\
\text { lesiones }\end{array}$ & Pulmón & Sacos aéreos & Riñón & Intestino \\
\hline T1 D0S1 H & Excelente & 1 & $\begin{array}{l}\text { Sin lesiones de } \\
\text { coloración } \\
\text { rosado }\end{array}$ & Transparentes & $\begin{array}{l}\text { Sin } \\
\text { lesiones }\end{array}$ & Normal
\end{tabular}




\begin{tabular}{|c|c|c|c|c|c|c|}
\hline T2 D0S2 M & Excelente & 1 & $\begin{array}{l}\text { Sin lesiones de } \\
\text { coloración } \\
\text { rosado }\end{array}$ & Transparentes & $\begin{array}{l}\text { Sin } \\
\text { lesiones }\end{array}$ & Normal \\
\hline T3 D1S1 H & Excelente & 1 & $\begin{array}{l}\text { Sin lesiones de } \\
\text { coloración } \\
\text { rosado }\end{array}$ & Transparentes & $\begin{array}{l}\text { Sin } \\
\text { lesiones }\end{array}$ & Normal \\
\hline T4 D1S2 M & Excelente & 1 & $\begin{array}{l}\text { Sin lesiones de } \\
\text { coloración } \\
\text { rosado }\end{array}$ & Transparentes & $\begin{array}{l}\text { Sin } \\
\text { lesiones }\end{array}$ & Normal \\
\hline T5 D2S1 H & Excelente & 1 & $\begin{array}{l}\text { Sin lesiones de } \\
\text { coloración } \\
\text { rosado }\end{array}$ & Transparentes & $\begin{array}{l}\text { Sin } \\
\text { lesiones }\end{array}$ & Normal \\
\hline T6 D2S2 M & Excelente & 1 & $\begin{array}{l}\text { Sin lesiones de } \\
\text { coloración } \\
\text { rosado }\end{array}$ & Transparentes & $\begin{array}{l}\text { Sin } \\
\text { lesiones }\end{array}$ & Normal \\
\hline T7 D3S1 H & Excelente & 1 & $\begin{array}{l}\text { Sin lesiones de } \\
\text { coloración } \\
\text { rosado }\end{array}$ & Transparentes & $\begin{array}{l}\text { Sin } \\
\text { lesiones }\end{array}$ & Normal \\
\hline T8 D3S2 M & Excelente & 1 & $\begin{array}{l}\text { Sin lesiones de } \\
\text { coloración } \\
\text { rosado }\end{array}$ & Transparentes & $\begin{array}{l}\text { Sin } \\
\text { lesiones }\end{array}$ & Normal \\
\hline
\end{tabular}

Elaboración propia.

Fuente: Investigación de campo.

Para el análisis del objetivo 3 se evaluó la rentabilidad de los costos de producción del ensayo desglosados por tratamiento se realizó el análisis económico a través del indicador beneficio/costo.

Cuadro 17. Relación beneficio costo entre tratamientos

\begin{tabular}{|c|c|c|c|c|c|c|c|c|c|}
\hline \multirow{3}{*}{$\begin{array}{l}\text { Costos } \\
\text { Concepto }\end{array}$} & \multirow[b]{3}{*}{ Unidad } & \multicolumn{8}{|c|}{ TRATAMIENTOS } \\
\hline & & \multirow{2}{*}{$\begin{array}{l}\text { T1 } \\
\text { V.T }\end{array}$} & \multirow{2}{*}{$\begin{array}{l}\mathrm{T} 2 \\
\mathrm{~V} . \mathrm{T} \\
\end{array}$} & \multirow{2}{*}{$\begin{array}{l}\text { T3 } \\
\text { V.T } \\
\end{array}$} & \multirow{2}{*}{$\begin{array}{l}\text { T4 } \\
\text { V.T }\end{array}$} & \multirow{2}{*}{$\begin{array}{l}\text { T5 } \\
\text { V.T }\end{array}$} & \multirow{2}{*}{$\begin{array}{l}\text { T6 } \\
\text { V.T } \\
\end{array}$} & \multirow{2}{*}{$\begin{array}{l}\text { T7 } \\
\text { V.T }\end{array}$} & \multirow{2}{*}{$\begin{array}{l}\text { T8 } \\
\text { V.T } \\
\end{array}$} \\
\hline & & & & & & & & & \\
\hline Animales & Pollos & 35,1 & 35,1 & 35,1 & 35,1 & 35,1 & 35,1 & 35,1 & 35,1 \\
\hline Alimento inicial & qq & 116 & 116 & 116 & 116 & 116 & 116 & 116 & 116 \\
\hline Alimento final & $\mathrm{qq}$ & 124,8 & 150,8 & 124,8 & 150,8 & 124,8 & 150,8 & 124,8 & 150,8 \\
\hline Vacunas & Unidad & 5 & 5 & 5 & 5 & 5 & 5 & 5 & 5 \\
\hline Gas & Tanques & 4 & 4 & 4 & 4 & 4 & 4 & 4 & 4 \\
\hline Tamo & $\mathrm{qq}$ & 10 & 10 & 10 & 10 & 10 & 10 & 10 & 10 \\
\hline Antibióticos & Gramos & 5 & 5 & 2 & 2 & 2 & 2 & 2 & 2 \\
\hline Ácido Orgánico & $\mathrm{ml}$ & 0 & 0 & 9,3 & 9,3 & 18,6 & 18,6 & 28 & 28 \\
\hline SUB TOTAL & & 299,9 & 325,9 & 306,2 & 332,2 & 315,5 & 341,5 & 324,9 & 350,9 \\
\hline
\end{tabular}


Karen Pilar Quijije Quiroz, Omelio E. Borroto Leal, Hilda Brigitte Quijije Quiroz...

\begin{tabular}{llllllllll}
\hline Ingresos & & & & & & & & & \\
peso pollos & & 681,48 & 735,84 & 663,48 & 767,52 & 686,16 & 774 & 665,64 & 738 \\
Precio venta & Libra & 0,7 & 0,7 & 0,7 & 0,7 & 0,7 & 0,7 & 0,7 & 0,7 \\
Precio total & & 477,04 & 515,09 & 464,44 & 537,26 & 480,31 & 541,8 & 465,95 & 516,6 \\
Ingreso neto & & 177,14 & 189,19 & 158,24 & 205,06 & 164,81 & 200,3 & 141,05 & 165,7 \\
\hline Relación B/C & & $\mathbf{1 , 5 9 0 7}$ & $\mathbf{1 , 5 8 0 5}$ & $\mathbf{1 , 5 1 6 8}$ & $\mathbf{1 , 6 1 7 3}$ & $\mathbf{1 , 5 2 2 4}$ & $\mathbf{1 , 5 8 6 5}$ & $\mathbf{1 , 4 3 4 1}$ & $\mathbf{1 , 4 7 2 2}$ \\
\hline
\end{tabular}

Elaboración propia.

Fuente: Investigación de campo.

Como se aprecia en el cuadro, el tratamiento 4, con 1,61 B/Ces el que presenta el mejor beneficio económico al productor, y el tratamiento 7 con 1,43 de relación $\mathrm{B} / \mathrm{C}$, es el que genera menos ingresos económicos. Es oportuno citar que el testigo T1, es el segundo en producir recursos, sin embargo es notoria la importancia económica de emplear ácidos orgánicos en la producción de pollos de engordes.

En lo referente al sexo, es notorio que los beneficios económicos los producen los machos, sin embargo a nivel de hembra, el mejor tratamiento fue el T1 con 1,59 de relación B/C, otorgando 1,5 dólares por cada dólar invertido.

\section{CONCLUSIONES}

En base a los resultados que se obtuvieron en la investigación, se concluyó que:

- Para los factores de consumo de alimentos, ganancia de peso, conversión alimenticia, mortalidad en pollos tratados en el agua de bebida con ácidos orgánicos y sin ácidos orgánicos se obtuvo diferencias estadísticas significativas, donde se observa que el mejor tratamiento para CA es el T4 sexo macho, GP es el T6 sexo macho, IC es el T3 sexo hembra y cuya mortalidad fue baja.

- Para el factor necropsia aviar no se encontró efecto negativo que genere carne de baja calidad, al contrario el uso o no de ácidos orgánicos en esta investigación indica que la carne es excelente, con un pulmón rosado, sacos aéreos transparentes, riñón sin lesiones e intestino normal.

- Finalmente la estimación económica indica que el tratamiento 4 en machos y el 5 en hembras, ambos con aplicación de 1 cc y 2 cc ácidos orgánicos respectivamente, son los tratamientos que generan mejores beneficios económicos en la producción de los pollos parrilleros.

\section{REFERÉNCIAS BIBLIOGRÁFICAS}

Gonzáles, A. (2013). Efecto de la suplementación de ácidos orgánicos sobre los parámetros productivos en pollos de engorde. Revista de Investigaciones Veterinarias del Perú, 6. 
Medina N. M., G. C. (2014). Desempeño productivo de pollos de engorde suplementados. Rev Fac Med Vet Zoot. 61(3): 270-283., 14.

Patoja, S. (2011). Evaluaciòn de acidificante orgánico en la crianza de pollos broiler en la provincia de Pichincha. Bolivar, Guaranda, Ecuador . obtenido de http://www.ammrveterinarios.com/ammrveterinarios/manuales/crianza\%20de\%20pollos $\% 20$ broiler.pdf

Rogel, J. L. (2014). "Evaluación de dos acidificantes comerciales en elrendimiento productivo de pollos camperos en elcantón Zapotillo". Obtenido de http://dspace.unl.edu.ec/jspui/bitstream/123456789/10602/1/trabajo\%20final\%20de\%20te sis\%20graduacion.pdf

Trujillo, M. P. (2016). Evaluación del desarrollo de pollos broiler mediante diferentes dosis de neutralizante de micotoxinas por procesos de biotransformación. Obtenido de http://dspace.ueb.edu.e:

http://dspace.ueb.edu.ec/bitstream/123456789/1504/1/Proyecto\%20de\%20Investigacion.p df 
Karen Pilar Quijije Quiroz, Omelio E. Borroto Leal, Hilda Brigitte Quijije Quiroz...

58 UNESUM-Ciencias. Publicación cuatrimestral. Vol. 3, Año 2019, No. 2 (Mayo - Agosto) 